\title{
Precision calculations for top-quark pair production at the LHC
}

\author{
loannis Tsinikos * \\ Centre for Cosmology, Particle Physics and Phenomenology (CP3), \\ Université Catholique de Louvain, Chemin du Cyclotron 2, B-1348 Louvain-la-Neuve, Belgium, \\ Technische Universität München, James-Franck-Str. 1, D-85748 Garching, Germany \\ E-mail: ioannis.tsinikos@uclouvain.be, ioannis.tsinikos@tum.de
}

In this proceeding we present precision calculation results for top-quark pair production at the LHC at NNLO QCD and NLO EW accuracy. Concerning the combination of QCD and EW corrections we discuss and adopt the multiplicative approach. We use and compare the latest available PDF sets to date, including the photon PDF, i.e. the LUXQED17 and the NNPDF3.1LUXQED. We find that the EW corrections are independent on the two PDF sets at differential level for all the main $t \bar{t}$ distributions. We further discus the size of the EW corrections w.r.t. the theory uncertainties and point out the reduction of the PDF uncertainties for the NNPDF3.1 PDF set.

XXVI International Workshop on Deep-Inelastic Scattering and Related Subjects (DIS2018) 16-20 April 2018

Kobe, Japan

${ }^{*}$ Speaker. Preprint: CP3-18-53, TUM-HEP-1154-18 


\section{Introduction}

In this proceeding we present methods and results, which are extensively discussed in [1] for the LHC and in [2] for the Tevatron. With the LHC running at $13 \mathrm{TeV}$ the experimental uncertainties will significantly decrease for the $t \bar{t}$ process. The tension between theory and experiment reported for the $p_{T}(t)$ distribution at $8 \mathrm{TeV}[3,4]$ will be examined in detail with the data at $13 \mathrm{TeV}$. Calculations in $t \bar{t}$ beyond the NLO in QCD are presented in [5-21]. The inclusion of EW corrections to $t \bar{t}$ production is realised in [22-34]. The complete NLO EW corrections require the photon-induced subprocesses. For this reason several PDF sets are developed including the photon PDF. These are the MRST2004QED [35], the NNPDF2.3QED [36], the APFEL_NN2.3QED [37,38], the CT14QED [39], the NNPDF3.0QED [40], the LUXQED [41] and the very recent LUXQED17 [42] and NNPDF3.1LUXQED [43,44]. For the $t \bar{t}$ production the NNPDF2.3QED and CT14QED PDF sets are compared in $[45,46]$ whereas the LUXQED and the NNPDF3.0QED ones in $[1,47]$. Similar comparisons between the NNPDFQED and the CT14QED, LUXQED PDF sets are realised for dilepton final states in [48,49].

\section{Calculation framework}

In this work we present a NNLO QCD + NLO EW calculation for $t \bar{t}$ production at differential level using the multiplicative combination. For the NNLO QCD part we use the calculational techniques of [8] and for the NLO EW part we use the currently public version of the MADGRAPH5_AMC@NLO framework [50,51] already validated in [52-54]. In figure 1 we present a pictorial representation of the perturbative orders considered in the calculation.

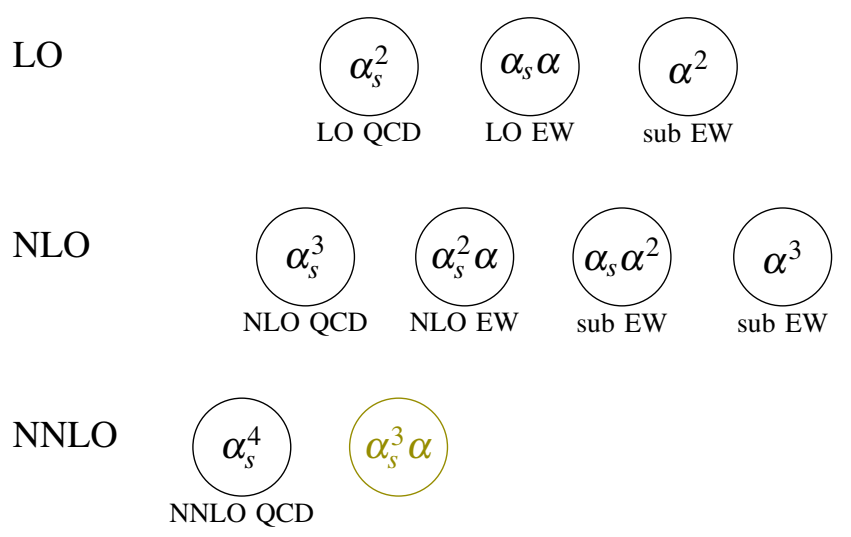

Figure 1: Perturbative orders included in the calculation (black-coloured=additive approach, +greencoloured=multiplicative approach).

The NNLO QCD corrections, as expected, reduce significantly the scale uncertainties and as a result the subleading $\alpha^{2}, \alpha_{s} \alpha^{2}, \alpha^{3}$ orders (sub EW) need to be included. In figure 1, the consideration of only the black coloured perturbative orders defines the additive approach. In this approach we 
define the QCD, EW and QCD+EW orders for an observable $\Sigma$ as follows:

$$
\begin{aligned}
& \Sigma_{\mathrm{QCD}} \equiv \Sigma_{\mathrm{LO} \mathrm{QCD}}+\Sigma_{\mathrm{NLO}} \mathrm{QCD}+\Sigma_{\mathrm{NNLO}} \mathrm{QCD} \\
& \Sigma_{\mathrm{EW}} \equiv \Sigma_{\mathrm{LO} \mathrm{EW}}+\Sigma_{\mathrm{NLO} \text { EW }}+\Sigma_{\text {sub EW }} \\
& \Sigma_{\mathrm{QCD}+\mathrm{EW}} \equiv \Sigma_{\mathrm{QCD}}+\Sigma_{\mathrm{EW}}
\end{aligned}
$$

Using the ingredients of equation 2.1 one can approximate the missing higher order mixed term $O\left(\alpha_{s}^{3} \alpha\right)$ (denoted with green colour in figure 1) by applying the NLO QCD K-factor on the $\alpha_{s}^{2} \alpha$ perturbative order. This defines the multiplicative approach as follows:

$$
\Sigma_{\mathrm{QCD} \times \mathrm{EW}} \equiv \Sigma_{\mathrm{QCD}+\mathrm{EW}}+\left(K_{\mathrm{QCD}}^{\mathrm{NLO}}-1\right) \Sigma_{\mathrm{NLO} \mathrm{EW}}
$$

Following the setup of [1], we use the 5-flavour scheme for the calculation and the EW parameters are defined in the $G_{\mu}$-scheme. In equation 2.3 we show the input parameters of the calculation.

$$
\begin{aligned}
m_{t} & =173.3 \mathrm{GeV}, m_{H}=125.09 \mathrm{GeV}, m_{W}=80.385 \mathrm{GeV}, m_{Z}=91.1876 \mathrm{GeV}, \\
G_{\mu} & =1.1663787 \cdot 10^{-5} \mathrm{GeV}^{-2}, \\
\mu & =\frac{m_{T, t}}{2} \text { for the } p_{T}(t) \text { distribution, } \mu=\frac{m_{T, \bar{t}}}{2} \text { for the } p_{T}(\bar{t}) \text { distribution, } \\
\mu & =\frac{H_{T}}{4}=\frac{1}{4}\left(m_{T, t}+m_{T, \bar{t}}\right) \text { for the } m(t \bar{t}), y_{\text {avt }}, y(t \bar{t}) \text { distributions. }
\end{aligned}
$$

The observables $p_{T, \text { avt }}, y_{\text {avt }}$ are defined as the average of the two top $p_{T}$ and $y$ distributions respectively. The particular scale choices are based on the principle of the fastest convergence, are taken from [8] and are supported by the recent calculation at NLO+NNLL' accuracy [20]. The scale uncertainties are calculated by the 7-point variation in the interval $\left\{\mu / 2<\mu_{f}, \mu_{r}<2 \mu\right\}$. In the following section we present results on the comparison between the NNPDF3.1LUXQED and LUXQED17 PDF sets at $13 \mathrm{TeV}$.

\section{Results}

In this section we will first show comparisons between the two PDF sets at differential level for $m(t \bar{t}), p_{T, \text { avt }}, y_{\text {avt }}$ and $y(t \bar{t})$ and then discuss specific distributions. In figure 2 we show the ratio
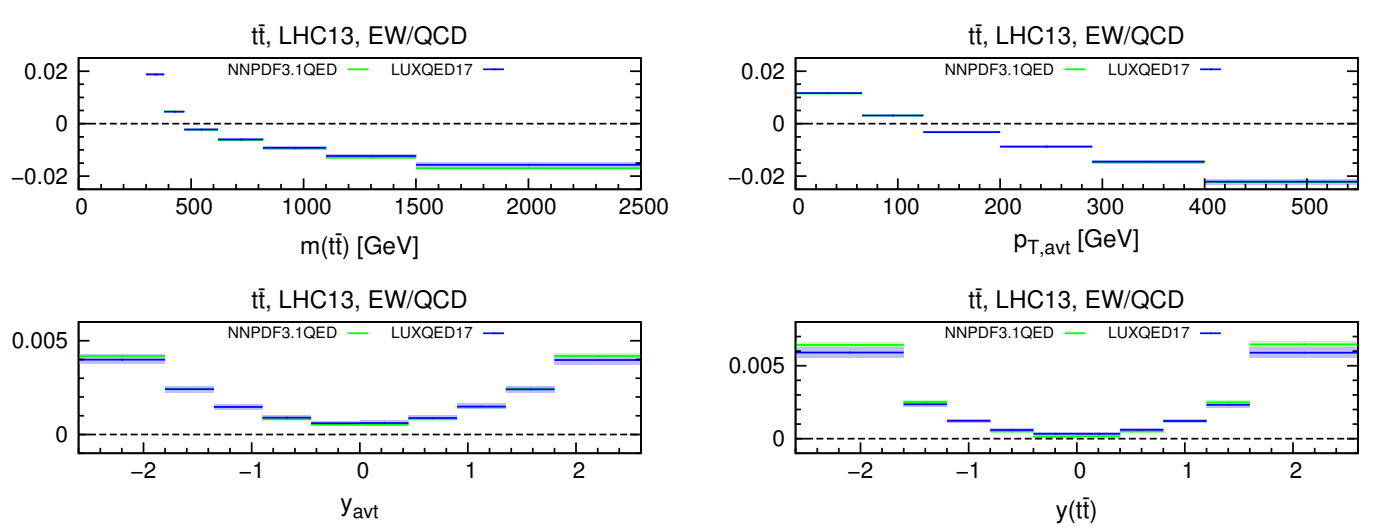

Figure 2: The impact of the EW corrections for the $m(t \bar{t}), p_{T, \text { avt }}, y_{\mathrm{avt}}, y_{t \bar{t}}$ distributions for the NNPDF3.1LUXQED and the LUXQED17 PDF sets. 
EW/QCD. In these ratio plots we can see that the two PDF sets are in very good agreement with each other. We remind to the reader that this was not the case in similar comparisons between the NNPDF3.0QED and the LUXQED PDF sets [1,47]. The NNPDF collaboration adopted the LUXQED approach for the photon PDF in the 3.1 version. Detailed comparisons among the aforementioned PDF sets are realised in $[43,44]$, two of which we show in figure 3.
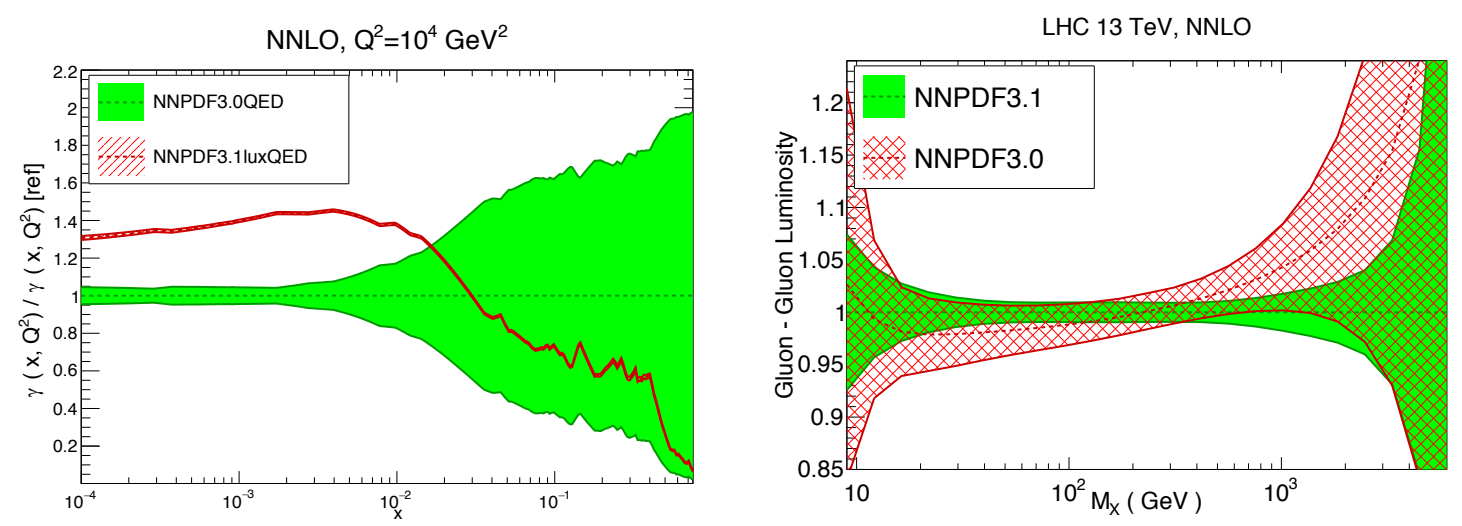

Figure 3: Comparisons between the NNPDF3.0 and the NNPDF3.1 PDF sets. The plots are taken from $[43,44]$.

In the left plot of figure 3 we can see the reduction on the uncertainty of the photon PDF for NNPDF3.1 as well as the reduction of the central value at the high $x$ region. In the right plot it is interesting to notice the reduction on the uncertainty on the $g g$ luminosity for the NNPDF3.1.
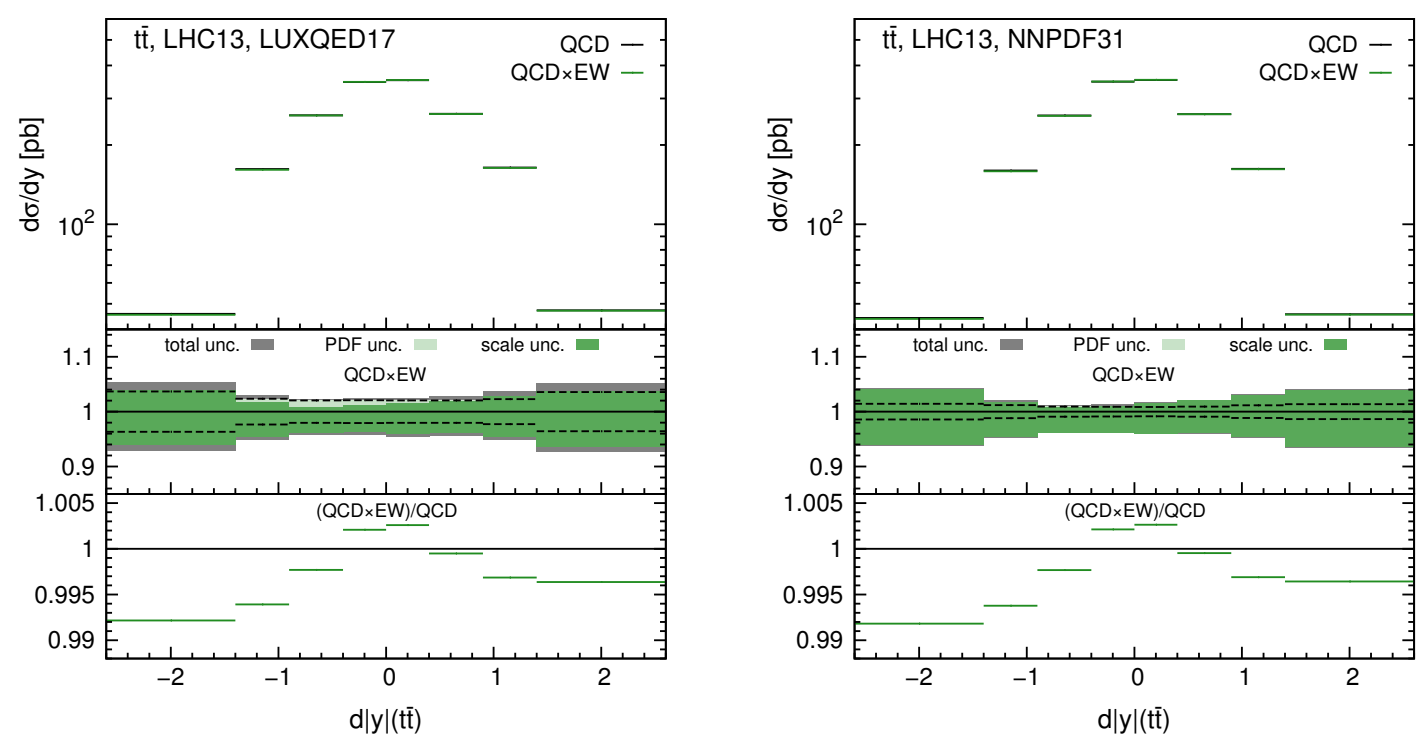

Figure 4: The $d|y|(t \bar{t})$ distribution at $13 \mathrm{TeV}$. The format of the plot is explained in the text.

Focusing on specific differential distributions at $13 \mathrm{TeV}$ we start with the $d|y|(t \bar{t}) \equiv|y(t)|-$ $|y(\bar{t})|$ in figure 4 . The format of the plots in figures 4 and 5 is the following. In the main panel we show the distributions of the QCD (black) and QCD $\times$ EW (green) according to the definitions 
of equations 2.1 and 2.2. In the first inset we show the scale (green) and PDF (light green-dashed black) uncertainties of the $\mathrm{QCD} \times \mathrm{EW}$ prediction as well as their sum in quadrature (grey). In the second inset we show the ratio $(\mathrm{QCD} \times \mathrm{EW}) / \mathrm{QCD}$ in order to separate the effect of the $\mathrm{EW}$ corrections. On the left plots we have the results with the LUXQED17 and on the right plots the ones with the NNPDF3.1LUXQED. The $d|y|(t \bar{t})$ distribution is the one on which the $t \bar{t}$ centralperipheral asymmetry $\left(A_{C}\right)$ is built. In the last inset we can see that the EW corrections are not symmetric around zero, which indicates their contribution to the asymmetry. A dedicated work on this observable at this level of accuracy is presented in [55] for both the total cross section and differential distributions. In figure 5 we show the $m(t \bar{t})$ and $p_{T, \text { avt }}$ results. In both these distributions we can see the sudakov logarithmic suppression rendered by the virtual EW corrections. With the
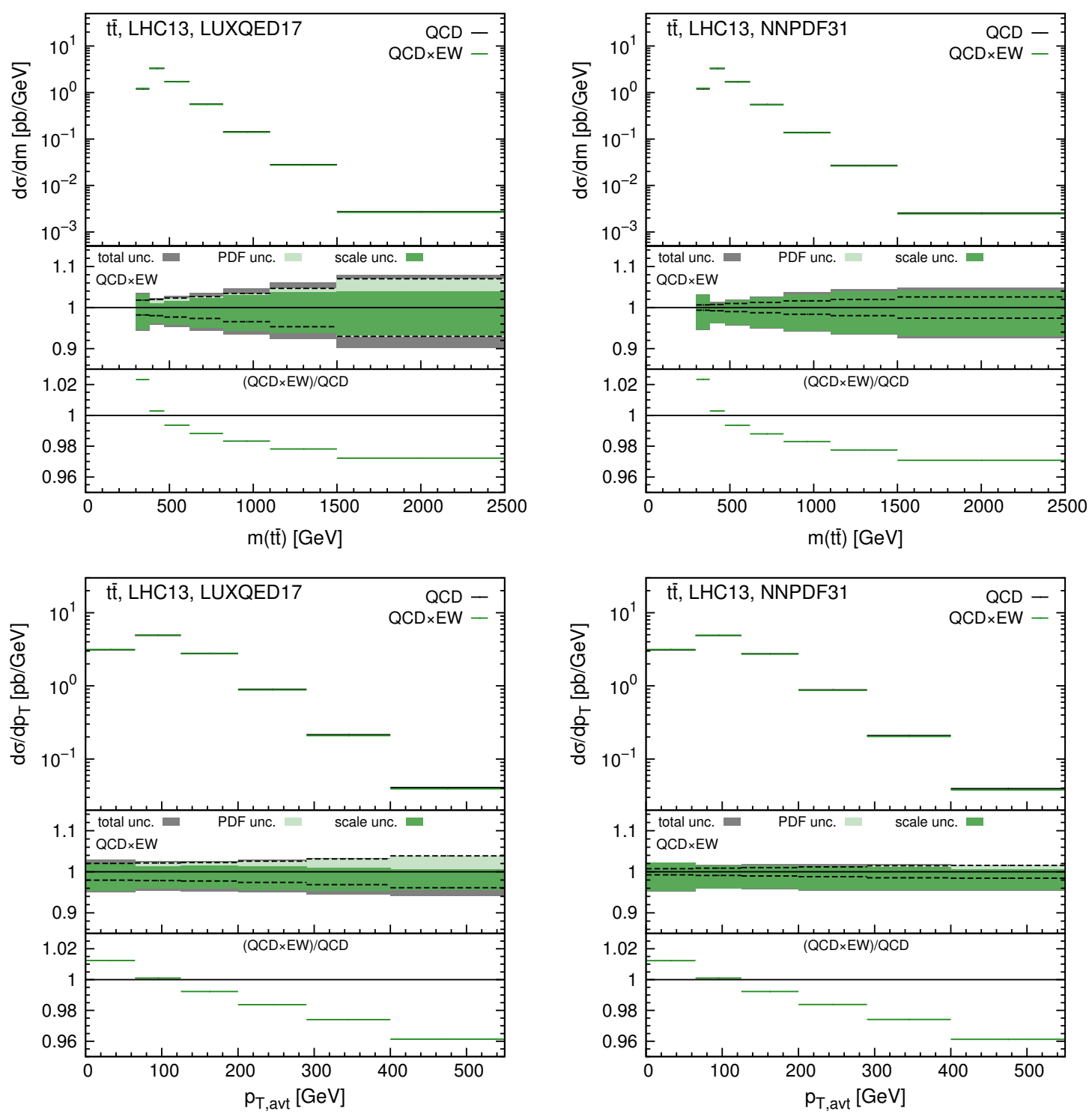

Figure 5: The $m(t \bar{t})$ (top) and $p_{T, \text { avt }}$ (bottom) distributions at $13 \mathrm{TeV}$. The format of the plots is explained in the text. 
photon PDF effect being small to negligible and since the Heavy Boson Radiation (HBR) is not included as describing different processes ${ }^{1}$, there is nothing to compensate this suppression at the high energy regime. Looking at the $m(t \bar{t})$ distribution in the second inset, we can see that in both PDF sets the EW corrections are below 4\% even at the region of $2 \mathrm{TeV}$ of invariant mass with the theory uncertainties (first inset) being of the order of $\sim 10 \%$ in that region. However, in the $p_{T, \text { avt }}$ distribution the situation is different. The EW corrections induce a similar maximum $4 \%$ effect but already at $p_{T} \sim 500 \mathrm{GeV}$. This is a regime where the theory uncertainties are of the order of $5 \%$ and furthermore experimentally will be measured very accurately at $13 \mathrm{TeV}$. In all cases in figures 4 and 5 we notice that the EW corrections are no longer sensitive to the choice of the PDF set, which is in agreement with what we see also in figure 2. Furthermore we notice that in the LUXQED17 (left) plots the PDF uncertainties are comparable with the scale ones $\left(d|y|(t \bar{t})\right.$ distribution) and especially in the tails of the $m(t \bar{t})$ and $p_{T \text {,avt }}$ distributions they become the dominant theory uncertainty. However this is not the case in the NNPDF3.1LUXQED (right) plots. This is mostly due to the reduction of the PDF uncertainties in the gg luminosity for the NNPDF3.1 PDF set, shown in the right plot of figure 3. A detailed discussion on this improvement is realised in $[43,44]$.

\section{Conclusions}

In this proceeding we show the calculation framework described in [1] and we apply it for the comparison of the latest available PDF sets which have QCD evolution at NNLO and include the photon PDF with the LUXQED approach. We explain and restrict ourselves to the multiplicative combination of the NNLO QCD and NLO EW corrections to $t \bar{t}$ distributions. We compare at differential level the results obtained with the LUXQED17 and NNPDF3.1LUXQED PDF sets. The results show that the inclusion of the NNLO QCD perturbative order reduces significantly the scale and therefore the total theory uncertainty. Furthermore we discuss the reduction of the PDF uncertainties in the NNPDF3.1LUXQED PDF set. The EW corrections are independent on the choice between the aforementioned two PDF sets. The effect of the EW corrections on the tail of the $p_{T, a v t}$ distribution is of the same order of the total theory uncertainty and in an accurately probed regime by the LHC run at $13 \mathrm{TeV}$. The distributions shown in this proceeding are part of the results produced to be compared with experimental data from CMS and are available in HTTP://WWW.PRECISION.HEP.PHY.CAM.AC.UK/RESULTS/TTBAR-NNLOQCD-NLOEW

\section{Acknowledgments}

I would like to thank M. Czakon, D. Heymes, A. Mitov, D. Pagani, M. Zaro and A. Papanastasiou for their collaboration on this work. My participation to this workshop was supported by the F.R.S.-FNRS "Fonds de la Recherche Scientifique" (Belgium). I would like also to thank the department of physics at TUM (Munich) for the hospitality during the completion of this project.

\footnotetext{
${ }^{1}$ It is shown explicitly in [1] that the inclusion of the HBR causes an effect always smaller in comparison to the EW corrections.
} 


\section{References}

[1] M. Czakon, D. Heymes, A. Mitov, D. Pagani, I. Tsinikos and M. Zaro, Top-pair production at the LHC through NNLO QCD and NLO EW, 1705.04105.

[2] M. Czakon, D. Heymes, A. Mitov, D. Pagani, I. Tsinikos and M. Zaro, Top-quark pair production at NNLO QCD + NLO EW accuracy: Tevatron results, in 10th International Workshop on Top Quark Physics (TOP2017) Braga, Portugal, September 17-22, 2017, 2017. 1712.04842.

[3] CMS collaboration, V. Khachatryan et al., Measurement of the differential cross section for top quark pair production in pp collisions at $\sqrt{s}=8 \mathrm{TeV}$, Eur. Phys. J. C75 (2015) 542, [1505.04480].

[4] ATLAS collaboration, G. Aad et al., Measurements of top-quark pair differential cross-sections in the lepton+jets channel in pp collisions at $\sqrt{s}=8 \mathrm{TeV}$ using the ATLAS detector, 1511.04716.

[5] M. Czakon, M. L. Mangano, A. Mitov and J. Rojo, Constraints on the gluon PDF from top quark pair production at hadron colliders, JHEP 07 (2013) 167, [1303. 7215].

[6] M. Czakon, P. Fiedler and A. Mitov, Total Top-Quark Pair-Production Cross Section at Hadron Colliders Through $O\left(\alpha_{S}^{4}\right)$, Phys. Rev. Lett. 110 (2013) 252004, [1303. 6254].

[7] M. Czakon, D. Heymes and A. Mitov, High-precision differential predictions for top-quark pairs at the LHC, Phys. Rev. Lett. 116 (2016) 082003, [1511. 00549 ].

[8] M. Czakon, D. Heymes and A. Mitov, Dynamical scales for multi-TeV top-pair production at the LHC, JHEP 04 (2017) 071, [1606.03350].

[9] S. Moch and P. Uwer, Theoretical status and prospects for top-quark pair production at hadron colliders, Phys. Rev. D78 (2008) 034003, [0 804 . 1476].

[10] N. Kidonakis, Two-loop soft anomalous dimensions and NNLL resummation for heavy quark production, Phys. Rev. Lett. 102 (2009) 232003, [0903.2561].

[11] M. Czakon, A. Mitov and G. F. Sterman, Threshold Resummation for Top-Pair Hadroproduction to Next-to-Next-to-Leading Log, Phys. Rev. D80 (2009) 074017, [0 907 . 1790 ].

[12] V. Ahrens, A. Ferroglia, M. Neubert, B. D. Pecjak and L. L. Yang, Renormalization-Group Improved Predictions for Top-Quark Pair Production at Hadron Colliders, JHEP 09 (2010) 097, [1003.5827].

[13] M. Beneke, P. Falgari and C. Schwinn, Threshold resummation for pair production of coloured heavy (s)particles at hadron colliders, Nucl. Phys. B842 (2011) 414-474, [1 007.5414$].$

[14] N. Kidonakis, Next-to-next-to-leading soft-gluon corrections for the top quark cross section and transverse momentum distribution, Phys. Rev. D82 (2010) 114030, [1009. 4935].

[15] V. Ahrens, A. Ferroglia, M. Neubert, B. D. Pecjak and L.-L. Yang, RG-improved single-particle inclusive cross sections and forward-backward asymmetry in $t \bar{t}$ production at hadron colliders, JHEP 09 (2011) 070, [1103.0550].

[16] V. Ahrens, A. Ferroglia, M. Neubert, B. D. Pecjak and L. L. Yang, Precision predictions for the $t+t$ (bar) production cross section at hadron colliders, Phys. Lett. B703 (2011) 135-141, [1105.5824].

[17] M. Beneke, P. Falgari, S. Klein and C. Schwinn, Hadronic top-quark pair production with NNLL threshold resummation, Nucl. Phys. B855 (2012) 695-741, [1109.1536]. 
[18] M. Cacciari, M. Czakon, M. Mangano, A. Mitov and P. Nason, Top-pair production at hadron colliders with next-to-next-to-leading logarithmic soft-gluon resummation, Phys. Lett. B710 (2012) 612-622, [1111.5869].

[19] B. D. Pecjak, D. J. Scott, X. Wang and L. L. Yang, Resummed differential cross sections for top-quark pairs at the LHC, 1601.07020.

[20] M. Czakon, A. Ferroglia, D. Heymes, A. Mitov, B. D. Pecjak, D. J. Scott et al., Resummation for (boosted) top-quark pair production at NNLO+NNLL' in QCD, JHEP 05 (2018) 149, [1803.07623].

[21] S. Catani, M. Grazzini and H. Sargsyan, Transverse-momentum resummation fo top-quark pair production at the LHC, 1806.01601.

[22] W. Beenakker, A. Denner, W. Hollik, R. Mertig, T. Sack and D. Wackeroth, Electroweak one loop contributions to top pair production in hadron colliders, Nucl. Phys. B411 (1994) 343-380.

[23] J. H. Kühn, A. Scharf and P. Uwer, Electroweak effects in top-quark pair production at hadron colliders, Eur. Phys. J. C51 (2007) 37-53, [hep-ph/ 0610335$].$

[24] J. H. Kühn, A. Scharf and P. Uwer, Weak Interactions in Top-Quark Pair Production at Hadron Colliders: An Update, Phys. Rev. D91 (2015) 014020, [1305. 5773].

[25] W. Bernreuther, M. Fuecker and Z. G. Si, Mixed QCD and weak corrections to top quark pair production at hadron colliders, Phys. Lett. B633 (2006) 54-60, [hep-ph/ 0508091$].$

[26] J. M. Campbell, D. Wackeroth and J. Zhou, Study of weak corrections to Drell-Yan, top-quark pair, and dijet production at high energies with MCFM, Phys. Rev. D94 (2016) 093009, [1608 . 03356 ].

[27] W. Hollik and D. Pagani, The electroweak contribution to the top quark forward-backward asymmetry at the Tevatron, Phys. Rev. D84 (2011) 093003, [1107.2606].

[28] J. H. Kühn and G. Rodrigo, Charge asymmetries of top quarks at hadron colliders revisited, JHEP 01 (2012) 063, [1109.6830].

[29] A. V. Manohar and M. Trott, Electroweak Sudakov Corrections and the Top Quark Forward-Backward Asymmetry, Phys. Lett. B711 (2012) 313-316, [1201. 3926].

[30] W. Bernreuther and Z.-G. Si, Top quark and leptonic charge asymmetries for the Tevatron and LHC, Phys. Rev. D86 (2012) 034026, [1205.6580].

[31] W. Bernreuther and Z.-G. Si, Distributions and correlations for top quark pair production and decay at the Tevatron and LHC., Nucl. Phys. B837 (2010) 90-121, [1003.3926].

[32] A. Denner and M. Pellen, NLO electroweak corrections to off-shell top-antitop production with leptonic decays at the LHC, JHEP 08 (2016) 155, [1607.05571].

[33] W. Hollik and M. Kollar, NLO QED contributions to top-pair production at hadron collider, Phys. Rev. D77 (2008) 014008, [0 708 . 1697].

[34] C. Gütschow, J. M. Lindert and M. Schönherr, Multi-jet merged top-pair production including electroweak corrections, Eur. Phys. J. C78 (2018) 317, [1803.00950].

[35] A. D. Martin, R. G. Roberts, W. J. Stirling and R. S. Thorne, Parton distributions incorporating QED contributions, Eur. Phys. J. C39 (2005) 155-161, [hep-ph/0 411040 ].

[36] NNPDF collaboration, R. D. Ball, V. Bertone, S. Carrazza, L. Del Debbio, S. Forte, A. Guffanti et al., Parton distributions with QED corrections, Nucl. Phys. B877 (2013) 290-320, [1308 . 0598 ]. 
[37] V. Bertone, S. Carrazza and J. Rojo, APFEL: A PDF Evolution Library with QED corrections, Comput. Phys. Commun. 185 (2014) 1647-1668, [1310 .1394].

[38] V. Bertone, S. Carrazza, D. Pagani and M. Zaro, On the Impact of Lepton PDFs, JHEP 11 (2015) 194, [1508.07002].

[39] C. Schmidt, J. Pumplin, D. Stump and C. P. Yuan, CTI4QED parton distribution functions from isolated photon production in deep inelastic scattering, Phys. Rev. D93 (2016) 114015, [1509.02905].

[40] NNPDF collaboration, R. D. Ball et al., Parton distributions for the LHC Run II, JHEP 04 (2015) 040, [1410.8849].

[41] A. Manohar, P. Nason, G. P. Salam and G. Zanderighi, How bright is the proton? A precise determination of the photon parton distribution function, Phys. Rev. Lett. 117 (2016) 242002, [1607.04266].

[42] A. V. Manohar, P. Nason, G. P. Salam and G. Zanderighi, The Photon Content of the Proton, JHEP 12 (2017) 046, [1708.01256].

[43] NNPDF collaboration, R. D. Ball et al., Parton distributions from high-precision collider data, Eur. Phys. J. C77 (2017) 663, [1706.00428].

[44] NNPDF collaboration, V. Bertone, S. Carrazza, N. P. Hartland and J. Rojo, Illuminating the photon content of the proton within a global PDF analysis, SciPost Phys. 5 (2018) 008, [1712. 07053].

[45] D. Pagani, I. Tsinikos and M. Zaro, The impact of the photon PDF and electroweak corrections on $t \bar{t}$ distributions, 1606.01915.

[46] I. Tsinikos, EW corrections to t $\bar{t}$ distributions: the photon PDF effect, in 9th International Workshop on Top Quark Physics (TOP 2016) Olomouc, Czech Republic, September 19-23, 2016, 2016. 1611.08234.

[47] I. Tsinikos, EW corrections on top-quark pair production: the impact of the photon PDF, PoS DIS2017 (2018) 138.

[48] E. Accomando, J. Fiaschi, F. Hautmann, S. Moretti and C. H. Shepherd-Themistocleous, The effect of real and virtual photons in the di-lepton channel at the LHC, Phys. Lett. B770 (2017) 1-7, [1612.08168].

[49] E. Accomando, J. Fiaschi, F. Hautmann, S. Moretti and C. H. Shepherd-Themistocleous, Real and virtual photons effects in di-lepton production at the LHC, in 21 st Rencontres de Physique de La Vallée d'Aoste: Rencontres de La Thuile (La Thuile) La Thuile, Aosta, Italy, March 5-11, 2017, 2017. 1704.08587.

[50] J. Alwall, R. Frederix, S. Frixione, V. Hirschi, F. Maltoni, O. Mattelaer et al., The automated computation of tree-level and next-to-leading order differential cross sections, and their matching to parton shower simulations, JHEP 07 (2014) 079, [1405.0301].

[51] R. Frederix, S. Frixione, V. Hirschi, D. Pagani, H. S. Shao and M. Zaro, The automation of next-to-leading order electroweak calculations, 1804.10017.

[52] S. Frixione, V. Hirschi, D. Pagani, H. S. Shao and M. Zaro, Electroweak and QCD corrections to top-pair hadroproduction in association with heavy bosons, JHEP 06 (2015) 184, [150 4.03446 ].

[53] J. R. Andersen et al., Les Houches 2015: Physics at TeV Colliders Standard Model Working Group Report, in 9th Les Houches Workshop on Physics at TeV Colliders (PhysTeV 2015) Les Houches, France, June 1-19, 2015, 2016. 1605.04692. 
[54] R. Frederix, S. Frixione, V. Hirschi, D. Pagani, H.-S. Shao and M. Zaro, The complete NLO corrections to dijet hadroproduction, JHEP 04 (2017) 076, [1612.065 48].

[55] M. Czakon, D. Heymes, A. Mitov, D. Pagani, I. Tsinikos and M. Zaro, Top-quark charge asymmetry at the LHC and Tevatron through NNLO QCD and NLO EW, Phys. Rev. D98 (2018) 014003, [1711.03945]. 